\title{
Silencing of long non-coding RNA NEAT1 inhibits hepatocellular carcinoma progression by downregulating SMO by sponging microRNA-503
}

\author{
CHUIHUA SUN $^{1}$, TING XIAO ${ }^{2}$, YING XIAO ${ }^{3}$ and YUNBAO $\mathrm{II}^{4}$ \\ Departments of ${ }^{1}$ Intensive Care Unit and ${ }^{2}$ Infectious Diseases, Weifang People's Hospital, Weifang, Shandong 261041; \\ ${ }^{3}$ Department of Ultrasound, 960 Hospital of The Chinese People's Liberation Army, Tai'an Hospital, Tai'an, Shandong 271000; \\ ${ }^{4}$ Department of Clinical Laboratory, Jinan Chain Medical Laboratory Co., Ltd., Jinan, Shandong 250000, P.R. China
}

Received September 6, 2019; Accepted November 13, 2020

DOI: $10.3892 / \mathrm{mmr} .2020 .11807$

\begin{abstract}
Hepatocellular carcinoma(HCC) poses an increasing threat to humans, due to its poor prognosis. Nuclear-enriched abundant transcript 1 (NEAT1), a type of long non-coding (lnc)RNA, has been found to function in a variety of cancer types. However, the role of NEAT1 in HCC is poorly understood. Reverse transcription-quantitative PCR was used to detect the expression levels of NEAT1, microRNA (miR)-503 and Smoothened (SMO) mRNA in HCC tissues and cells. MTT and flow cytometry assays were used to investigate cell viability and apoptosis, respectively, while Transwell assays were performed to investigate cell invasion and migration. StarBase and TargetScan were utilized to predict the target sequence between miR-503 and NEAT1 or SMO, the results of which were verified using a dual-luciferase reporter assay. The protein expression level of SMO was measured using western blot. The RNA expression level of NEAT1 and SMO was significantly elevated in HCC tissues and cells compared with that in the corresponding healthy tissues and cells, which was contrary to miR-503 expression level. NEAT1 silencing was found to restrict the viability, migration and invasion of the cells, while simultaneously induced apoptosis in the HCC cell line. Further studies found that miR-503 expression was negatively correlated with NEAT1 or SMO. It was also confirmed that NEAT1 directly interacted with miR-503 and miR-503 could bind to the 3'-untranslated region of SMO. Furthermore, overexpression of NEAT1 or SMO could reverse the effects of miR-503-mediated inhibition on cell viability, invasion, migration and promotion of apoptosis in the HCC
\end{abstract}

Correspondence to: Mrs. Ting Xiao, Department of Infectious Diseases, Weifang People's Hospital, 151 Guangwen, Kuiwen, Weifang, Shandong 261041, P.R. China

E-mail: xiaoting7047@163.com

Key words: hepatocellular carcinoma, long non-coding RNA nuclear-enriched abundant transcript 1 , apoptosis, microRNA-503, smoothened cell lines. These results demonstrated that downregulation of NEAT1 impeded the viability, migration, invasion and induced apoptosis through the NEAT1/miR-503/SMO axis in the HCC cell line.

\section{Introduction}

Hepatocellular carcinoma (HCC) is a primary liver cancer, caused by cirrhosis resulting from infection with a Hepatitis virus, typically Hepatitis B or C, or alcohol (1). In 2012, 782,000 individuals suffered from liver cancer, and in 2015 HCC resulted in 810,500 cancer-associated deaths worldwide (2). The high incidence and mortality rates indicate that it is imperative to identify new therapeutic methods and molecular targets for HCC treatment.

Long non-coding (lnc)RNAs are a class of RNA molecules, $>200$ nucleotides in length, which do not encode proteins (3). Unusual expression of lncRNAs often appears in different types of cancer (4-6), including $\mathrm{HCC}(7,8)$. Accumulating data demonstrated that IncRNAs played a central role in cancer initiation, progression and growth in multiple tumors (9-11). The lncRNA, nuclear-enriched abundant transcript 1 (NEAT1), is specifically localized in nuclear paraspeckles (12). It was reported to be abnormally, highly expressed in glioma and lung cancer, but was lowly expressed in de novo acute promyelocytic leukemia (13-15). Zhu et al (16) found that NEAT1 was upregulated in HCC and promoted the malignant biological properties of HCC. However, the mechanism of NEAT1 in regulating $\mathrm{HCC}$ requires further investigation.

MicroRNAs (miRNAs/miRs) are highly conserved and small non-coding RNAs ( 22 nucleotides in length), which regulate gene expression levels by specifically targeting the 3'-untranslated regions (3'-UTR) of mRNA at the post-transcriptional level (17). Research into different types of cancer have increased the understanding of the role of miRNAs (18-20). Several studies have confirmed that miR-503 could serve as a tumor suppressor in HCC $(21,22)$. However, the precise mechanism of miR-503 has not been completely elucidated.

Smoothened, frizzled class receptor (SMO) is a class of frizzled $G$ protein-coupled receptor. Increasing evidence has indicated that SMO participated in the regulation of numerous 
types of cancer, including basal-cell carcinomas and breast cancer, and it might function as an oncogene $(23,24)$. However, whether SMO could be an attractive cancer drug target requires further investigation.

In the present study, the expression levels of NEAT1 were investigated in HCC tissues and cells. In addition, the function of NEAT1 and the regulatory mechanism between NEAT1, miR-503 and SMO in HCC progression were also further investigated.

\section{Materials and methods}

Samples and cell culture. Hepatocellular carcinoma tissues and adjacent normal tissues $(>5 \mathrm{~cm}$ laterally from the edge of the cancerous region) were collected from 31 patients with HCC (<43 age $\leq 67 ; 19$ male and 12 female patients) during surgeries between July 2016 and January 2019 at Weifang People's Hospital (Shandong, China). The included patients did not receive any chemotherapy or radiation treatment prior to surgery. All patients provided written informed consent and the present study was authorized by the Ethics Committee of Weifang People's Hospital (Shandong, China).

All cell lines (the human normal hepatic cell line, QZG, and the HCC cell lines, Huh-7 and Hep3B) were purchased from The Cell Bank of Type Culture Collection of the Chinese Academy of Sciences. Dulbecco's modified Eagle's medium, supplemented with $10 \%$ fetal bovine serum (FBS) (both from Sigma-Aldrich; Merck KGaA), was used to culture the cells at $37^{\circ} \mathrm{C}$ in a humidified incubator with $5 \% \mathrm{CO}_{2}$.

Cell transfection. Small interfering (si) RNA targeting NEAT1 (si-NEAT1), miR-503 mimic (miR-503) and its inhibitor (anti-miR-503), as well as the corresponding negative controls, were purchased from Shanghai GenePharma Co., Ltd. The sequences for siRNAs and miRNAs were as follows: si-NEAT1, 5'-UGGUAAUGGUGGAGGAAGAUU-3'; si-NC, 5'-UUCUCC GAACGUGUCACGU-3'; miR-503 mimic, 5'-UAGCAGCGG GAACAGUUCUGCAG-3'; miR-NC mimic, 5'-UCACAACCU CCUAGAAAGAGUAGA-3'; anti-miR-503, 5'-UAGCAGCGG GAACAGUUCUGCAG-3'; and anti-miR-NC, 5'-UUGUAC UACACAAAAGUACUG-3'. The full-length NEAT1 cDNA and the 3'UTR of SMO were synthesized by Guangzhou RiboBio Co., Ltd., and cloned into the vector, pcDNA3.1 (Invitrogen; Thermo Fisher Scientific, Inc.). These were subsequently verified using sequencing (conducted by Guangzhou RiboBio Co., Ltd.), named as pcDNA-NEAT1 and pcDNA-SMO, and the empty vector was used as a negative control (pcDNA-control). When cells were cultured to $\sim 70 \%$ confluence, the siRNAs (final concentration, $20 \mathrm{nM}$ ), miRNAs (at final concentration of $20 \mathrm{nM}$ for miRNA mimic, and $25 \mathrm{nM}$ for miRNA inhibitor) and vectors (at final concentration of $1 \mu \mathrm{g} / \mathrm{ml}$ ) were transfected into the cells using Lipofectamine ${ }^{\circledR} 2000$ (Invitrogen; Thermo Fisher Scientific, Inc.) according to the manufacturer's protocol. Then, the cells were incubated in a humidified incubator with $5 \% \mathrm{CO}_{2}$ at $37^{\circ} \mathrm{C}$ for 24 or $48 \mathrm{~h}$ upon transfection. Cells were collected for further research after this incubation.

RNA isolation and reverse transcription-quantitative $P C R(R T-q P C R)$. Total RNA from the tissues or cells were isolated using a RNAiso Plus kit (Takara Biotechnology
Co., Ltd.) according to the manufacturer's instructions. After the concentration and purity of RNA were detected using a NanoDrop 2000/2000c (Thermo Fisher Scientific, Inc.), $1 \mu \mathrm{g}$ RNA was reverse transcribed into cDNA using the PrimeScript $^{\mathrm{TM}}$ RT Master Mix kit (Takara Biotechnology Co., Ltd.) at $37^{\circ} \mathrm{C}$ for $15 \mathrm{~min}$ and for a further $5 \mathrm{sec}$ at $85^{\circ} \mathrm{C}$. The RT-qPCR was conducted using a Fast SYBR ${ }^{\circledR}$ Green Master Mix kit (Thermo Fisher Scientific, Inc.) using the following thermocycling conditions: Initial denaturation at $95^{\circ} \mathrm{C}$ for $10 \mathrm{~min}$ followed by 35 cycles of $95^{\circ} \mathrm{C}$ for $15 \mathrm{sec}$ and $60^{\circ} \mathrm{C}$ for $1 \mathrm{~min}$. The data were analyzed using the $2^{-\Delta \Delta \mathrm{Cq}}$ method (25). $\beta$-actin and U6 were used as the endogenous controls. The primer sequences used are shown in Table I.

MTT assay. MTT (Sigma-Aldrich; Merck KGaA) was used to investigate cell viability. Transfected cells were seeded in 96 -well plates $\left(5 \times 10^{3}\right.$ cells per well) for $0,24,48$ or $72 \mathrm{~h}$. A total of $20 \mu \mathrm{l}$ MTT solution was added to the wells and the cells were incubated at $37^{\circ} \mathrm{C}$ for another $4 \mathrm{~h}$. Following which, the media was aspirated off and $200 \mu \mathrm{l}$ dimethyl sulfoxide (Thermo Fisher Scientific, Inc.) was added. Optical density values were examined at $490 \mathrm{~nm}$ using a microplate reader (Bio-Rad Laboratories, Inc.) and were analyzed with Microplate Manager Software v. 6.0 (Bio-Rad Laboratories, Inc.).

Cell migration and invasion assays. Transwell chambers, that were precoated with or without Matrigel at room temperature for $1 \mathrm{~h}$ (Corning Inc.) prior to use, were utilized to detect the invasion or migration abilities of the cells. Transfected cells in serum-free media were added to the upper chamber, and the lower chamber contained the media with $10 \%$ FBS. After incubation at $37^{\circ} \mathrm{C}$ for $24 \mathrm{~h}$, the cells on the upper surface of the membrane were wiped off with a cotton swab and the cells in lower surface were analyzed using an inverted microscope (Leica DMi1; Leica Microsystems, Inc.) at x40 magnification, following staining with crystal violet for $10 \mathrm{~min}$ at $37^{\circ} \mathrm{C}$. The upper chambers, coated with Matrigel (Corning Inc.), were used to determine cell invasion with the same method.

Apoptosis assay. Apoptosis was investigated using an Apoptosis Detection kit (BD Biosciences) according to the manufacturer's instructions. Cells were centrifuged at $200 \mathrm{x}$ g for $5 \mathrm{~min}$ at room temperature,resuspended using the provided binding buffer. Then, $5 \mu \mathrm{l}$ Annexin V-fluorescein isothiocyanate (Annexin V-FITC) and $5 \mu \mathrm{l}$ propidium iodide (PI) were added to the buffer in the dark for a further 5-min incubation at $4^{\circ} \mathrm{C}$. Stained cells were analyzed using an Attune NxT flow cytometer and Attune NxT Software version 3.2.1 (Thermo Fisher Scientific, Inc.).

Luciferase reporter assay. The target sites were analyzed using StarBase online software version 3.0 (between NEAT1 and miR-503) (26) or TargetScanHuman 7.1 (between SMO and miR-503) (27). The sequences of wild-type NEAT1 (WT-NEAT1), mutant NEAT1 (MUT-NEAT1), WT SMO 3'-UTR (WT-SMO) and MUT SMO 3'-UTR (MUT-SMO) containing the putative binding sites of miR-503 were amplified and ligated into the pGL3 luciferase reporter vector (Promega Corporation). The reporter vector and miR-503 or miR-control were cotransfected into the Huh-7 and Hep3B cells using Lipofectamine ${ }^{\circledR} 2000$ (Invitrogen; Thermo Fisher Scientific, 
Table I. Primer sequences used in RT-qPCR.

\begin{tabular}{|c|c|c|}
\hline Gene name & Forward (5'-3') & Reverse (5'-3') \\
\hline NEAT1 & TGGCTAGCTCAGGGCTTCAG & TCTCCTTGCCAAGCTTCСТTC \\
\hline miR-503 & ССТАТTTСССАТGАTТССТTСАТА & GTAATACGGTTATCCACGCG \\
\hline SMO & TGCTCATCGTGGGAGGCTACTT & ATCTTGCTGGCAGCCTTCTCAC \\
\hline$\beta$-actin & GCACCACACCTTCTACAATG & TGCTTGCTGATCCACATCTG \\
\hline U6 & CTTCGGCAGCACATATACT & AAAATATGGAACGCTTCACG \\
\hline
\end{tabular}

SMO, smoothened, frizzled class receptor; miR, microRNA; NEAT1, nuclear-enriched abundant transcript 1.
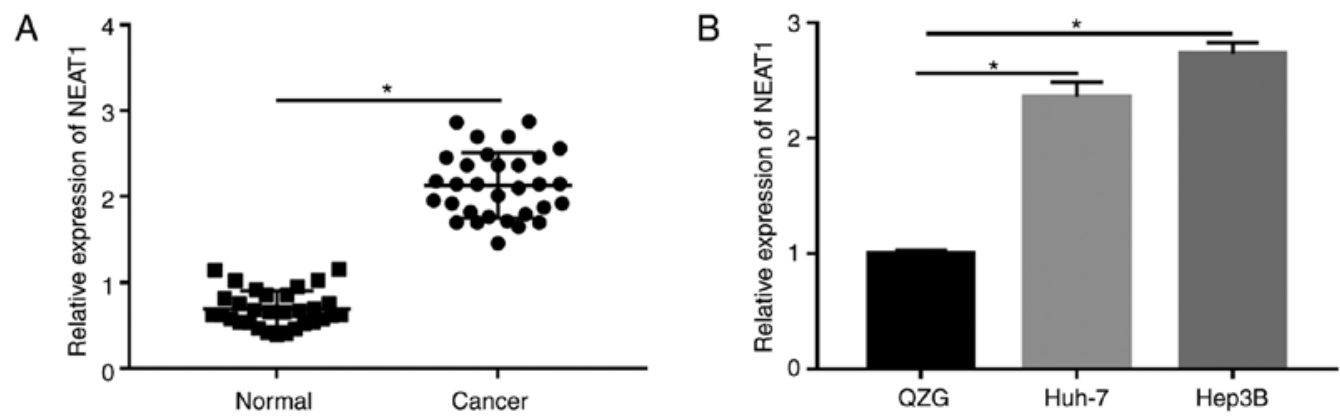

Figure 1. NEAT1 is significantly upregulated in HCC tissues and cells. (A) The expression level of NEAT1 was determined in (A) HCC ( $=31$ ) and adjacent normal tissues $(\mathrm{n}=31)$ and in (B) the non-malignant human epithelioid hepatic cell line (QZG) and the hepatocellular carcinoma cell lines (Huh-7 and Hep3B) using reverse transcription-quantitative PCR. "P<0.05. HCC, hepatocellular carcinoma; NEAT1, nuclear-enriched abundant transcript 1.

Inc.) as aforementioned. After $24 \mathrm{~h}$ of transfection, the luciferase activity was measured using the Dual-Glo ${ }^{\circledR}$ Luciferase Assay system (Promega Corporation). The firefly luciferase activity was normalized to the Renilla luciferase activity.

Western blot analysis. Total protein was extracted using the RIPA buffer (Thermo Fisher Scientific, Inc.) from tissues and cells. The protein concentration was determined using a Pierce $^{\mathrm{TM}}$ Rapid Gold BCA Protein Assay kit (Thermo Fisher Scientific, Inc.). Equal amounts of total protein (30 $\mu \mathrm{g} / \mathrm{lane})$ was separated using SDS-PAGE (10\% gels) then, transferred onto PVD membranes (Merck KGaA). Subsequently, the membranes were blocked with $5 \%$ skimmed milk for $3 \mathrm{~h}$ at $4^{\circ} \mathrm{C}$. After being washed three times with PBS, the membranes were incubated with the primary antibodies against SMO (1:1,000; cat. no. ab235183) or $\beta$-actin (1:2,500; cat. no. ab8226) (both from Abcam) overnight at $4^{\circ} \mathrm{C}$. The secondary antibody Goat Anti-Rabbit IgG H\&L [horseradish peroxidase (HRP)] (1:3,000; cat. no. ab205718; Abcam) and Goat Anti-Mouse IgG H\&L (HRP) (1:10,000; cat. no. ab205718; Abcam) were added for incubation for another $2 \mathrm{~h}$ at room temperature after being washed three times with PBS at room temperature. The protein bands were visualized using Super ECL Detection Reagent (Vazyme Biotech Co., Ltd.) and the ChemiDoc ${ }^{\mathrm{TM}} \mathrm{MP}$ Imaging System (Bio-Rad Laboratories, Inc.). ImageJ software V1.8.0_172 (National Institutes of Health) was used for semi-quantification of western blotting results.

Statistical analysis. Experimental data was analyzed using GraphPad Prism v7 software (GraphPad Software, Inc.) and are presented using the mean \pm standard deviation (SD). A total of 2 independent groups were compared using a paired or unpaired Student's t-test, while $>2$ groups were analyzed using the one-way ANOVA followed by Tukey's post hoc test. Every experiment was repeated three times, independently. The correlation between the expression levels of miR-503 and NEAT1 or SMO was analyzed using Pearson correlation analysis. $\mathrm{P}<0.05$ was considered to indicate a statistically significant difference.

\section{Results}

NEAT1 lncRNA was significantly increased in HCC tissues and cells. To investigate the role of NEAT1 in HCC, the mRNA expression level was first measured using RT-qPCR in HCC tissues. The data showed that NEAT1 was significantly upregulated in HCC tissues compared with that in the corresponding normal tissues (Fig. 1A). Subsequently, the mRNA expression levels of NEAT1 in the HCC cell lines Huh-7 and Hep3B were determined and the results revealed that NEAT1 expression was also highly elevated (Fig. 1B). These results supported the hypothesis that NEAT1 might function as an oncogene in the progression of HCC.

NEAT1 silencing induces apoptosis and inhibits the viability, migration and invasion of HCC cells. To further investigate the function of NEAT1 in HCC, the Huh-7 and Hep3B cell lines were transfected with si-NEAT1 and the transfection efficiency was determined using RT-qPCR (Fig. 2A). MTT assays revealed that the cell viability of the Huh-7 and Hep3B cell lines were both significantly suppressed in the si-NEAT1 group compared with that in the si-control group (Fig. 2B). 


\section{A}
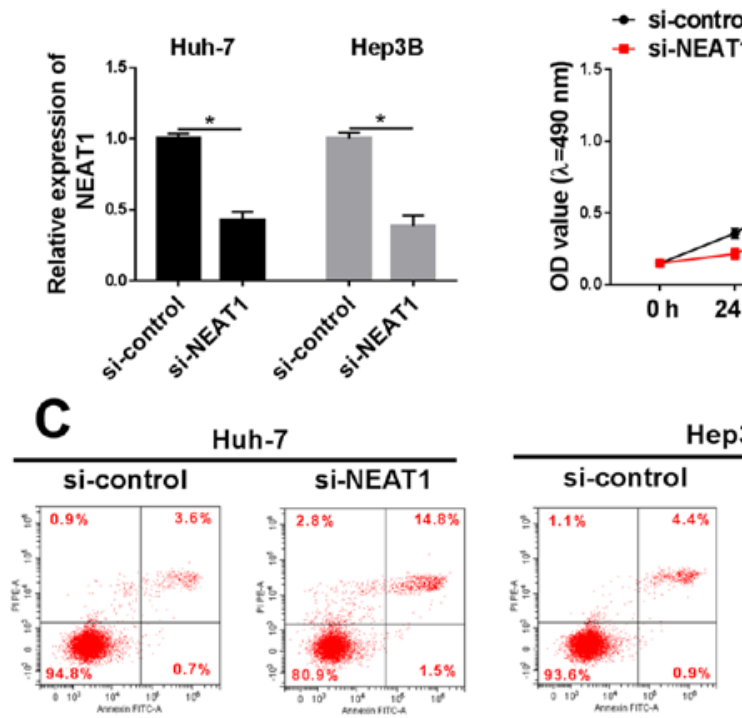

Нер3B

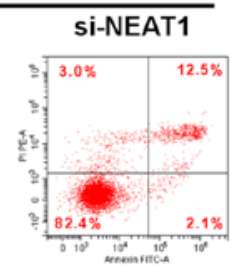

D
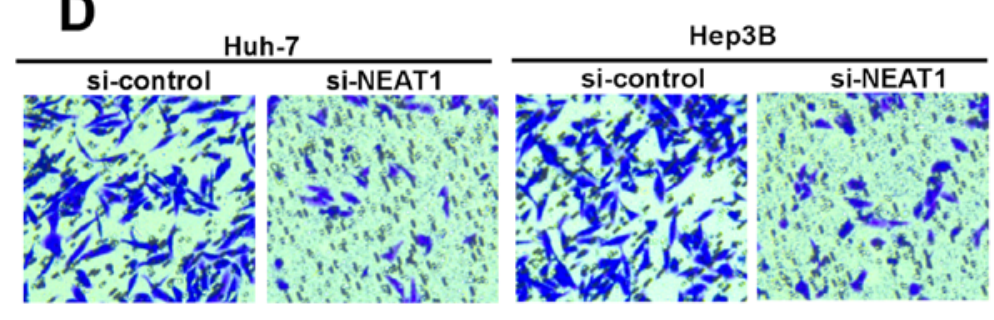

B

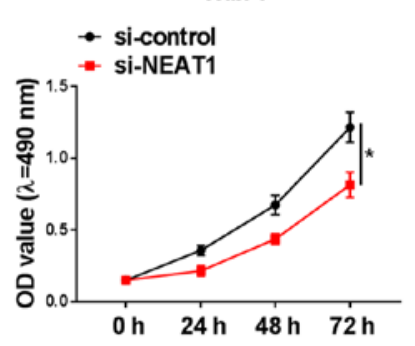

E
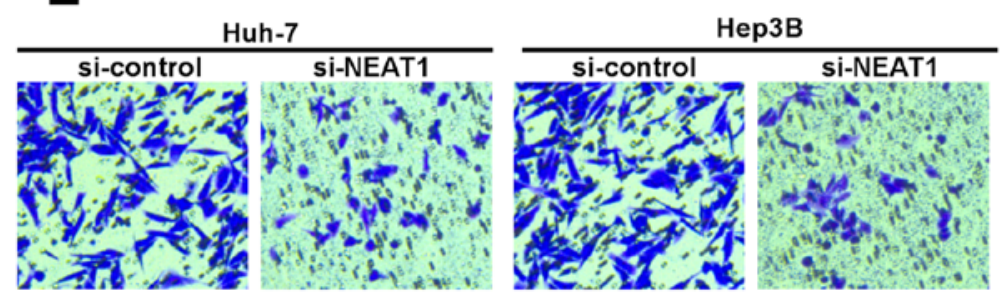
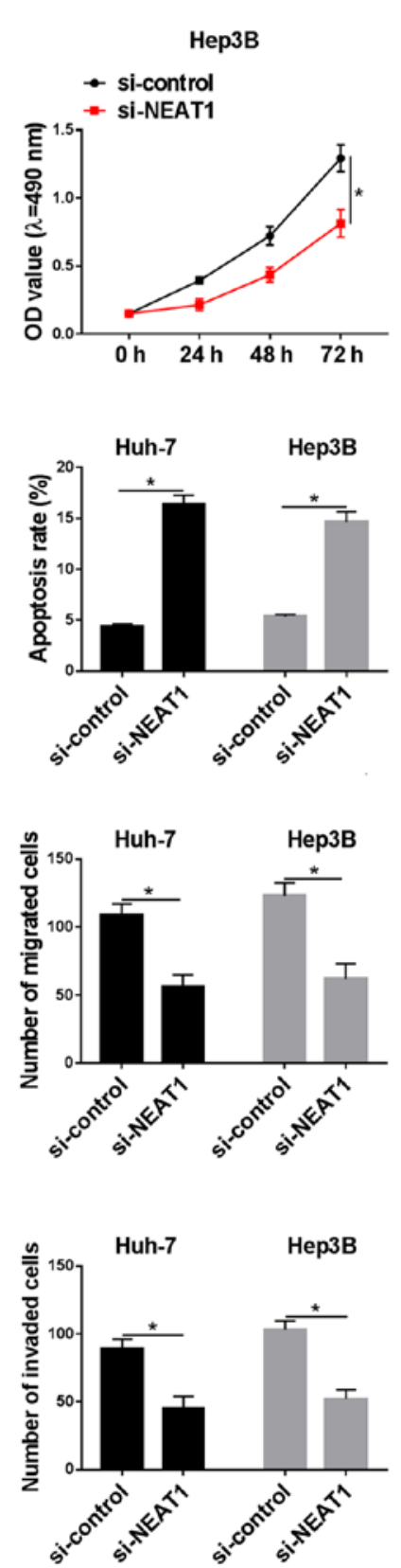

Figure 2. Silencing of NEAT1 inhibits cell viability, migration and invasion, and promoted apoptosis in hepatocellular carcinoma cells. Following transfection with si-NEAT1 or si-control, (A) the expression level of NEAT1, (B) cell viability and (C) apoptosis was investigated in the Huh-7 and Hep3B cell lines using reverse transcription-quantitative PCR, MTT assay and flow cytometry, respectively. Transwell (D) migration and (E) invasion assays were performed, and the numbers of migrated or invaded cells were calculated, following transfection with si-NEAT1 or si-control (magnification, $\mathrm{x} 100)$. "P<0.05. NEAT1, nuclear-enriched abundant transcript 1; si, small interfering RNA; OD, optical density.

Flow cytometry analyses of apoptosis demonstrated that knockdown of NEAT1 caused a significant increase in the rate of apoptosis in the two cell lines (Fig. 2C). Furthermore, Transwell assay, with or without Matrigel, indicated that downregulation of NEAT1 weakened the ability of HCC cells to invade or migrate (Fig. 2D and E). Taken together, these data demonstrated that NEAT1 silencing promoted cell apoptosis and suppressed the viability, migration and invasion of $\mathrm{HCC}$ cells in vitro.

NEAT1 directly interacts with miR-503 and negatively regulates the expression level of miR-503. Previous studies have confirmed that the interaction between IncRNA and miRNA has an essential role in the regulation of cancer in general $(28,29)$. Using the online database StarBase, NEAT1 was found to contain binding sites for miR-503 (Fig. 3A), and dual-luciferase reporter assays were conducted to validate the prediction. The results showed that the luciferase activity of WT-NEAT1 was significantly decreased by miR-503 in the Huh-7 and Hep3B cell lines, whereas the activity of MUT-NEAT1 was not changed (Fig. 3B). In addition, the expression level of miR-503 was significantly downregulated in tumor tissues (Fig. 3C) and the mRNA expression level of NEAT1 was negatively correlated with the expression of miR-503 in 31 HCC tissues (Fig. 3D). Furthermore, miR-503 expression was also found to be significantly decreased in the Huh-7 and Hep3B cell lines 

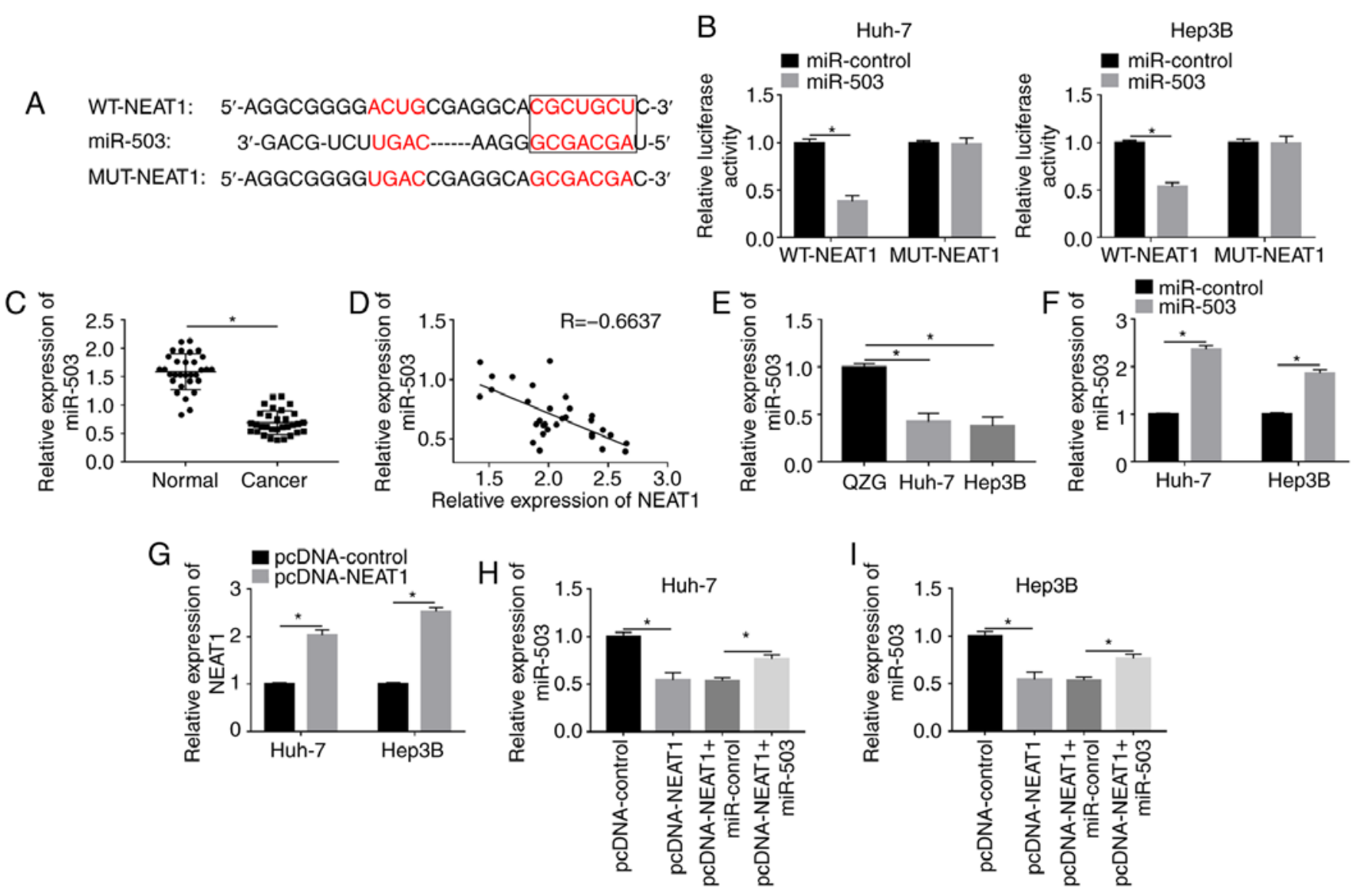

Figure 3. NEAT1 directly interacts with miR-503 and negatively regulates the expression level of miR-503. (A) Interaction between NEAT1 and miR-503 was predicted by StarBase online tool. (B) A dual-luciferase reporter assay was used to investigate the luciferase activity of cells cotransfected with miR-503 mimic and WT-NEAT1 or MUT-NEAT1 in both cell lines (C) The expression level of miR-503 in HCC tissues ( $\mathrm{n}=31$ ) and adjacent normal tissues ( $\mathrm{n}=31$ ) was measured using RT-qPCR. (D) Correlation between the expression level of NEAT1 and miR-503 in HCC tissues was analyzed. The expression level of miR-503 was detected using RT-qPCR in (E) QZG, Huh-7 and Hep3B cells and (F) Huh-7 and Hep3B cells transfected with miR-503 using RT-qPCR. (G) NEAT1 expression level in HCC cells transfected with pcDNA-control or pcDNA-NEAT1 was detected using RT-qPCR. miR-503 expression level in (H) Huh-7 and (I) Hep3B HCC cells transfected with pcDNA-NEAT1 or pcDNA-NEAT1 + miR-503 and their matched controls were determined using RT-qPCR. *P $<0.05$. NEAT1, nuclear-enriched abundant transcript 1; miR, microRNA; WT, wild-type; MUT, mutant; RT-qPCR, reverse transcription-quantitative PCR; HCC, hepatocellular carcinoma; miR-503, miR-503 mimic.

compared with that in the normal liver cell line (Fig. 3E). Next, miR-503 mimic and pcDNA-NEAT1 were used to overexpress miR-503 and NEAT1, respectively, and the expression levels of miR-503 and NEAT1 in the transfected cells were measured using RT-qPCR (Fig. 3F and G). The results revealed that upregulation of NEAT1 reduced the expression of miR-503, which could be rescued by enhancing miR-503 expression in HCC cells (Fig. 3H and I). The results illustrated that NEAT1 could interact with miR-503 and negatively modulated the expression level of miR-503 in the HCC cell lines.

NEAT1 overexpression reverses the miR-503-mediated effects on the viability, apoptosis, migration and invasion of the HCC cells. To further investigate the functions of the interaction between NEAT1 and miR-503 in the HCC cell lines, MTT, Transwell and apoptosis assays were performed. MTT assays indicated that overexpression of miR-503 reduced the viability of HCC cells compared with the miR-control group, whereas elevating the expression level of NEAT1 recovered cell viability and rescued the effect of miR-503-mediated inhibition on cell viability (Fig. 4A). Flow cytometry analysis of apoptosis demonstrated that NEAT1 overexpression reversed the effect of miR-503-mediated promotion on apoptosis (Figs. 4B; S1A and B). Similarly, Transwell assays revealed that miR-503 suppressed the migration and invasion of the HCC cell lines; however, the effects could be abrogated by NEAT1 overexpression (Figs. 4C and D; S1C-F). Collectively, these results suggested that upregulation of NEAT1 abolished the effects of miR-503-mediated inhibition on the viability, migration, invasion and promoted apoptosis of the HCC cell lines.

miR-503 targets and negatively regulates SMO expression level in vitro. To determine the underlying mechanism of miR-503 in HCCs, the TargetScan online tool was utilized to identify the possible target genes of miR-503. The result indicated that miR-503 had a potential binding site on the 3'-UTR of SMO (Fig. 5A). To validate the prediction, luciferase reporter plasmids harboring miR-503 targeting region (WT-SMO) and its mutant (MUT-SMO) were constructed. The results showed that miR-503 could reduce the luciferase activity of WT-SMO in the Huh and Hep3B cell lines, while it had little effect on the luciferase activity of MUT-SMO (Fig. 5B). In addition, SMO was found to be significantly upregulated in HCC tissues compared with that in the normal adjacent tissues 

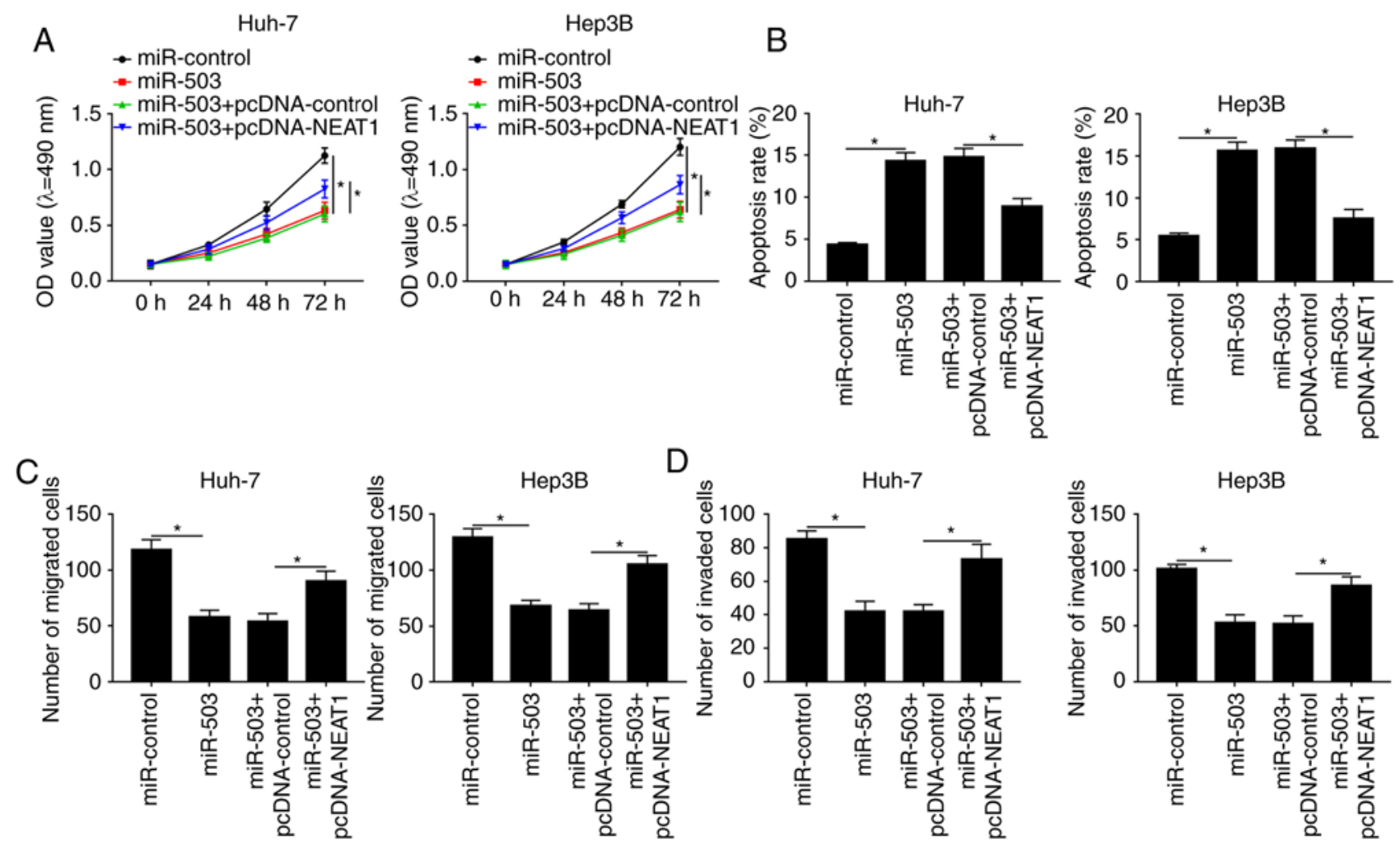

Figure 4. NEAT1 modulates hepatocellular carcinoma progression by targeting miR-503 in vitro. (A) Cell viability of the Huh-7 and Hep3B cells transfected with miR-503 or miR-503 + pcDNA-NEAT1 and corresponding controls was measured using MTT assays at different time points. (B) Flow cytometry was used to investigate apoptosis in the two cell lines. Transwell assays were conducted to determine cell (C) migration and (D) invasion. ${ }^{*} \mathrm{P}<0.05$. miR, microRNA; NEAT1, nuclear-enriched abundant transcript 1; OD, optical density.

(Fig. 5C). Further analysis indicated that the expression level of SMO was negatively correlated with miR-503 expression level (Fig. 5D), and the protein and mRNA expression levels of SMO were elevated in both HCC tissues and cells, respectively (Fig. 5E-G). Subsequently, the expression level of miR-503 in transfected HCC cells was investigated and the results showed that miR-503 was significantly reduced in HCC cells transfected with the miRNA inhibitor (anti-miR-503) compared with that in the control group (anti-control) (Fig. 5H). To investigate the association between SMO and miR-503 in the HCC cell lines, the expression level of SMO in HCC cells transfected with miR-503 or anti-miR-503, as well as with the corresponding controls, was measured. The results revealed that downregulation of miR-503 significantly increased the mRNA and protein expression levels of SMO in the HCC cell lines (Fig. 5I-L), which are also consistent with the finding that SMO mRNA expression level was negatively correlated with miR-503 expression level. Collectively, these results demonstrated that miR-503 could bind to the 3'-UTR of SMO and negatively modulated the expression level of SMO in the HCC cell lines.

SMO overexpression reverses the miR-503-mediated effects on viability, apoptosis, migration and invasion of the HCC cell lines. The association between SMO and miR-503 was further investigated with respect to HCC progression. MTT assays indicated that SMO overexpression reduced the effects of miR-503-mediated suppression on the viability of the HCC cell lines (Fig. 6A). Further analysis indicated that the miR-503-mediated promotion on apoptosis in the HCC cell lines was alleviated by the overexpression of SMO (Figs. 6B; S2A and B). Similarly, the miR-508-mediated inhibition of migration and invasion of the HCC cells could also be reduced by SMO overexpression (Figs. 6C and D; S2C-F). Collectively, these results indicated that miR-503 may serve as a tumor suppressor and SMO could function as an oncogene in the progression of HCC.

NEAT1 regulates SMO by sponging miR-503 in the HCC cells. As NEAT1 could directly interact with miR-503, and miR-503 could target the 3'-UTR of SMO, the underlying interactions between these nucleic acids were further investigated. The mRNA and protein expression levels of SMO in the Huh-7 and Hep3B cell lines transfected with miR-503 or miR-503 + pcDNA-NEAT1 were measured using RT-qPCR and western blot analysis, respectively (Fig. 7A-D). The results suggested that miR-503 overexpression significantly reduced SMO expression level. However, the suppression could be rescued following the transfection of pcDNA-NEAT1. These results indicated that NEAT1 might act as a competitive endogenous sponge, interacting with and downregulating miR-503, which affected the translational inhibition of SMO, which was targeted by miR-503.

\section{Discussion}

HCC is one of the leading causes of cancer-associated deaths worldwide $(2,30)$. Numerous studies have investigated the molecular mechanisms of HCC; however, further investigation 
WT-SMO-3'UTR: 5'-CUAACCCCCCUCCCCU CGCUGCUG-3' miR-503: 5'-GACGUCUUGACAAGG GCGCCGAU-3' MUT-SMO-3'UTR:5'-CUAACCCCCCUCCCCUAAUGAUGG-3'
B

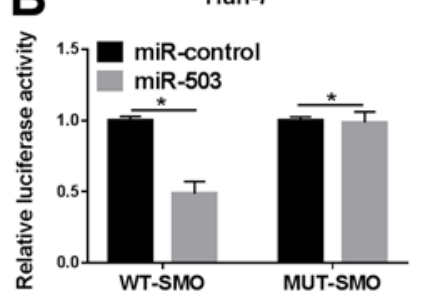

E
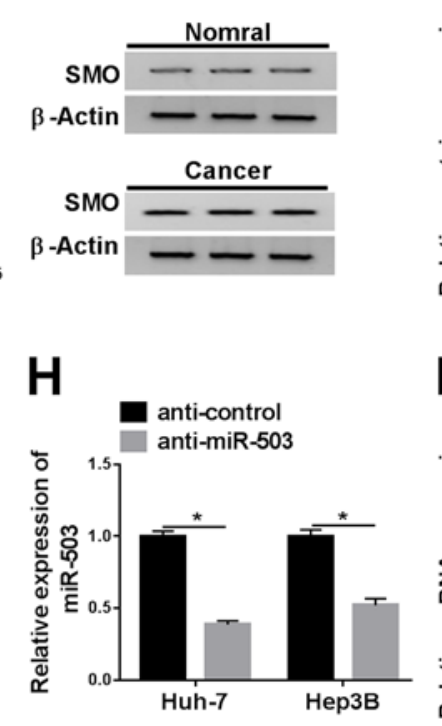

L

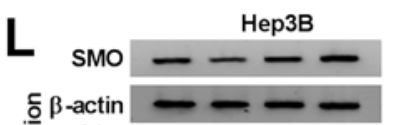

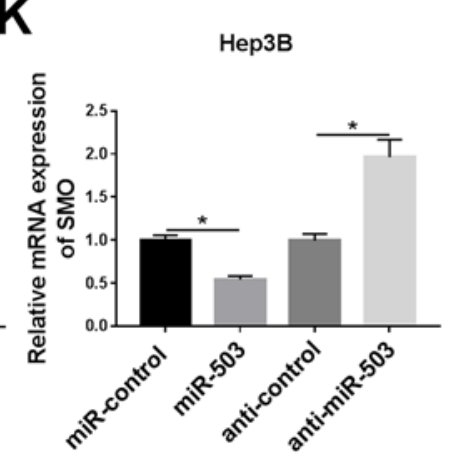
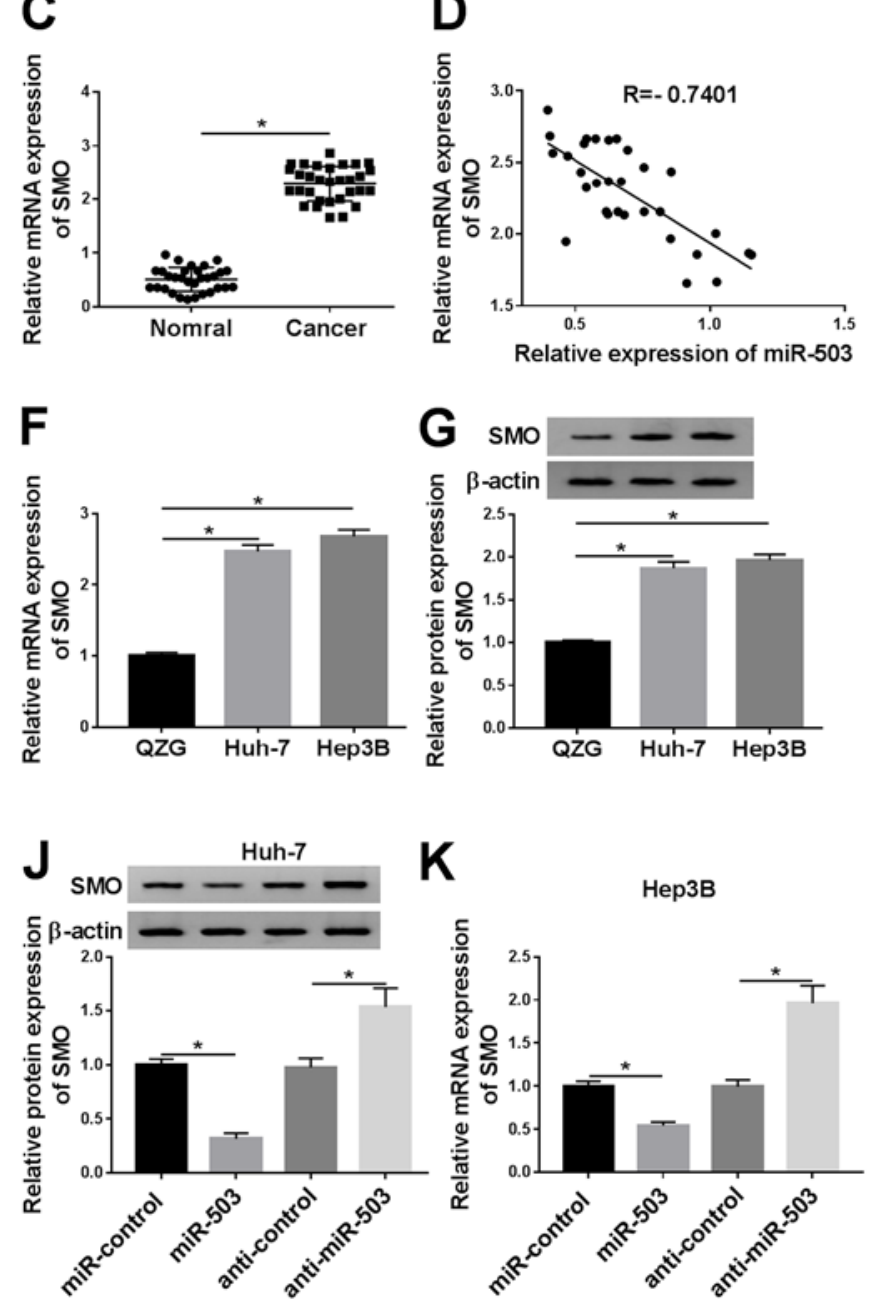
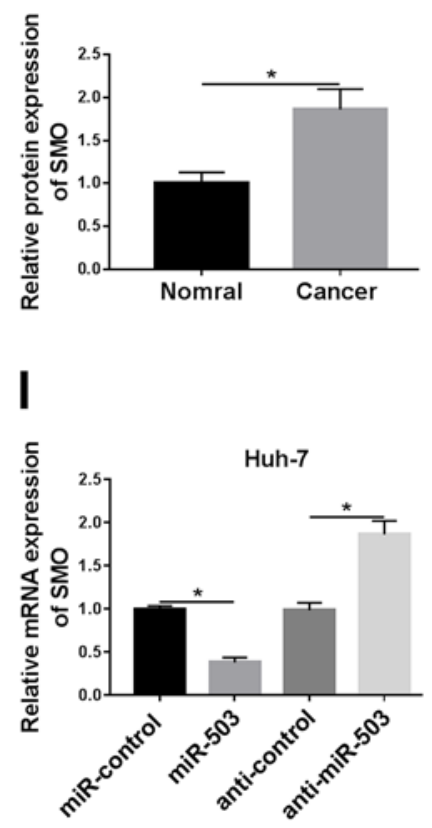

Figure 5. miR-503 binds to SMO and negatively regulates the expression level of SMO. (A) The binding sites between miR-503 and SMO were predicted using the TargetScan online tool. (B) The luciferase activity of cells cotransfected with the miR-503 and WT-SMO or MUT-SMO was investigated using a dual-luciferase assay. (C) The expression level of SMO in HCC tissues ( $\mathrm{n}=31)$ and adjacent normal tissues (n=31) was measured using RT-qPCR. (D) The correlation between the expression of SMO and miR-503 in HCC tissues was determined. (E) The protein expression level of SMO was detected using western blot analysis. The (F) mRNA and (G) protein expression levels of SMO were evaluated using RT-qPCR and western blot analysis, respectively. (H) The expression level of miR-503 in the Huh-7 and Hep3B cells transfected with anti-control or anti-miR-503 were detected using RT-qPCR. The mRNA and protein expression levels of SMO in (I and J) Huh-7 and (K and L) Hep3B HCC cells transfected with miR-503 or anti-miR-503 and their matched controls were determined using RT-qPCR and western blot analysis, respectively. ${ }^{*} \mathrm{P}<0.05$. WT, wild-type; MUT, mutant; RT-qPCR, reverse transcription-quantitative PCR; SMO, smoothened, frizzled class receptor; miR, microRNA; anti-miR-503, microRNA inhibitor; anti-control, microRNA inhibitor control; HCC, hepatocellular carcinoma.

is required to develop more effective treatments. The present study aimed to investigate the underlying mechanism of NEAT1 in the regulation of HCC progression.

Unusual expression of IncRNAs has been found in different types of cancer and has been associated with cancer progression $(4,6,13)$. Previous studies have indicated that the expression level of the lncRNA NEAT1 was significantly elevated in numerous types of human cancer, including non-small cell lung cancer, hepatocellular carcinoma and cervical cancer $(15,16,31)$, while Zhu et al (16) found that upregulated NEAT1 expression level promoted the malignant biological properties of HCC. The results of the present study are consistent with the study by Zhu et al (16), as it was observed that NEAT1 was markedly upregulated in HCC tissues compared with that in adjacent normal tissues, which indicated the potential importance of NEAT1 in the progression of HCC. In 

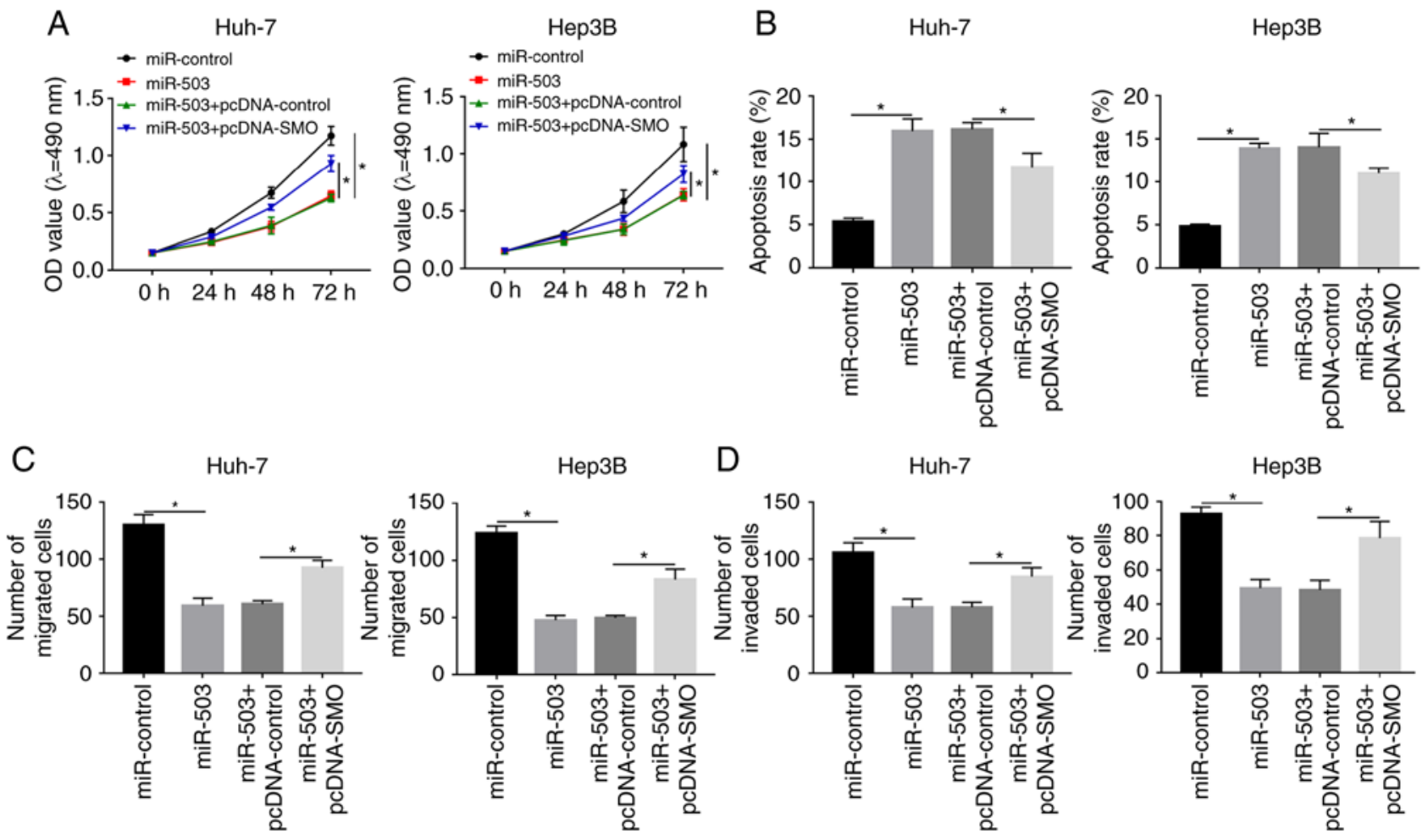

Figure 6. miR-503 modulates HCC progression by targeting SMO in vitro. (A) MTT assay was performed to determine the viability of the HCC cells transfected with miR-503 or miR-503 + pcDNA-SMO at different time points. (B) Flow cytometry was used to detect apoptosis in the HCC cells. (C) Cell migration and (D) invasion was performed using Transwell assays and the corresponding cell numbers were counted. ${ }^{*} \mathrm{P}<0.05$. miR, microRNA; HCC, hepatocellular carcinoma; SMO, smoothened, frizzled class receptor.
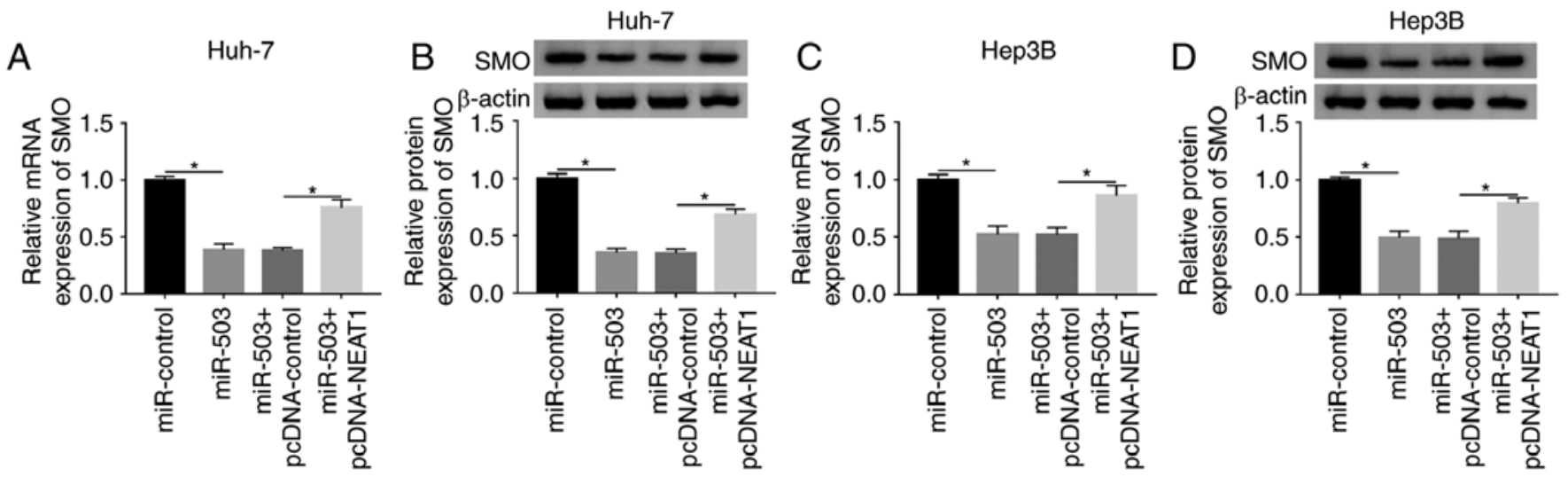

Figure 7. NEAT1 regulates SMO by sponging miR-503 in the HCC cells. The (A) mRNA and (B) protein expression of SMO in the Huh-7 cell line, transfected with miR-503 or miR-503 + pcDNA-NEAT1 was determined using RT-qPCR and western blot analysis, respectively. The (C) mRNA and (D) protein expression levels of SMO in the Hep3B cell line transfected with miR-503 or miR-503 + pcDNA-NEAT1were measured using RT-qPCR and western blot analysis, respectively. ${ }^{*} \mathrm{P}<0.05$. miR, microRNA; SMO, smoothened, frizzled class receptor; RT-qPCR, reverse transcription-quantitative PCR.

addition, further results from the present study demonstrated that NEAT1 silencing could reduce cell viability, migration and invasion and promoted apoptosis in the HCC cell lines. These results suggested that NEAT1 played an oncogenic role in the progression of HCC.

Previous studies have indicated that lncRNAs may serve as sponges to interact with miRNAs, thereby regulating cancer development $(28,29)$. Results from the present study identified miR-503 as a target of NEAT1. miR-503 has been previously reported to be reduced in HCC tissues, and inhibited cell proliferation and promoted apoptosis (22), the present study found the same results and also revealed that the expression level of miR-503 was also notably downregulated in 31 HCC tissues. Furthermore, the expression level of miR-503 was negatively correlated with NEAT1 in HCC tissues, and miR-503 was found to be downregulated by NEAT1. miR-503 overexpression suppressed cell viability, migration, invasion and induced apoptosis of the HCC cells, whereas its effects could be restricted by the overexpression of NEAT1. From these results, it was hypothesized that NEAT1 modulated HCC progression by targeting miR-503 in vitro. 
SMO was been found to be abnormally, highly expressed in numerous types of cancer, including sporadic basal-cell carcinoma and breast cancer, and might function as an oncogene $(23,24,32)$. A previous report indicated that SMO was upregulated in pancreatic cancer (33) and the results in the present study revealed that SMO was also markedly increased in HCC tissues and cells. Notably, SMO was predicted to be targeted by miR-503, and the interaction was subsequently validated using a dual-luciferase assay. Further analysis showed that miR-503 silencing increased the expression level of SMO, which clarified the negative correlation between SMO and miR-503. In addition, SMO overexpression could rescue the effects of miR-503-mediated suppression on cell viability, migration and invasion, and promoted the apoptosis of the HCC cells, which indicated that there was a tumor-promoting effect of SMO on HCC. The results from the present study suggested that miR-503 targeted the 3'-UTR of SMO in vitro and negatively regulated SMO expression in the progression of HCC.

As NEAT1 directly interacted with miR-503, and miR-503 could target the 3'-UTR of SMO, the potential mechanisms were further investigated. The results demonstrated that enhancing the expression level of miR-503 led to a significant reduction in SMO expression, however, the suppression was reduced by the subsequent overexpression of NEAT1. The results indicated that NEAT1 could act as a competitive endogenous sponge, which impaired the translational inhibition of SMO by interacting with and downregulating miR-503. Taken together, to the best of our knowledge, the present study has elucidated a new mechanism that NEAT1 regulated SMO by sponging miR-503 in HCC cells.

In summary, the present study revealed that NEAT1 and SMO were significantly elevated in HCC tissues and cells. In addition, NEAT1 could directly interact miR-503, which could target the 3'-UTR of SMO. Furthermore, downregulation of NEAT1 expression impaired cell viability, migration and invasion, and induced the apoptosis of HCC cells through the NEAT1/miR-503/SMO axis. The identification of the new NEAT1/miR-503/SMO axis might facilitate the development of novel therapeutic approaches for HCC.

\section{Acknowledgements}

Not applicable.

\section{Funding}

No funding was received.

\section{Availability of data and materials}

The datasets used and/or analyzed during the current study are available from the corresponding author on reasonable request.

\section{Authors' contributions}

CS conceived and designed the experiments. TX and YL performed the experiments. YX analyzed the data and wrote the manuscript. All authors read and approved the final manuscript.

\section{Ethics approval and consent to participate}

All patients provided written informed consent and this research was authorized by the Ethics Committee of Weifang People's Hospital (Shandong, China).

\section{Patient consent for publication}

Not applicable.

\section{Competing interests}

The authors declare that they have no competing interests.

\section{References}

1. GBD 2016 Causes of Death Collaborators: Global, regional, and national age-sex specific mortality for 264 causes of death, 1980-2016: A systematic analysis for the Global Burden of Disease Study 2016. Lancet 390: 1151-1210, 2017.

2. GBD 2015 Mortality and Causes of Death Collaborators: Global, regional, and national life expectancy, all-cause mortality, and cause-specific mortality for 249 causes of death, 1980-2015: A systematic analysis for the Global Burden of Disease Study 2015. Lancet 388: 1459-1544, 2016.

3. Mercer TR, Dinger ME and Mattick JS: Long non-coding RNAs: Insights into functions. Nat Rev Genet 10: 155-159, 2009.

4. Han P, Li JW, Zhang BM, Lv JC, Li YM, Gu XY, Yu ZW, Jia YH, Bai XF, Li L, et al: The lncRNA CRNDE promotes colorectal cancer cell proliferation and chemoresistance via miR-181a-5p-mediated regulation of Wnt/ $\beta$-catenin signaling. Mol Cancer 16: 9, 2017.

5. Hao NB,He YF,LiXQ,Wang K and Wang RL: The role of miRNA and lncRNA in gastric cancer. Oncotarget 8: 81572-81582, 2017.

6. Niknafs YS, Han S, Ma T, Speers C, Zhang C, Wilder-Romans K, Iyer MK, Pitchiaya S, Malik R, Hosono Y, et al: The lncRNA landscape of breast cancer reveals a role for DSCAM-AS1 in breast cancer progression. Nat Commun 7: 12791, 2016.

7. Li X, Wang S, Li Z, Long X, Guo Z, Zhang G, Zu J, Chen Y and Wen L: The lncRNA NEAT1 facilitates cell growth and invasion via the miR-211/HMGA2 axis in breast cancer. Int J Biol Macromol 105: 346-353, 2017.

8. Yu X, Li Z, Zheng H, Chan MT and Wu WK: NEAT1: A novel cancer-related long non-coding RNA. Cell Prolif 50: e12329, 2017.

9. Raveh E, Matouk IJ, Gilon M and Hochberg A: The H19 Long non-coding RNA in cancer initiation, progression and metastasis-a proposed unifying theory. Mol Cancer 14: 184, 2015.

10. Xie H, Liao X, Chen Z, Fang Y, He A, Zhong Y, Gao Q, Xiao H, Li J, Huang W and Liu Y: LncRNA MALAT1 inhibits apoptosis and promotes invasion by antagonizing miR-125b in bladder cancer cells. J Cancer 8: 3803-3811, 2017.

11. Yang G, Lu X and Yuan L: LncRNA: A link between RNA and cancer. Biochim Biophys Acta 1839: 1097-1109, 2014

12. Clemson CM, Hutchinson JN, Sara SA, Ensminger AW, Fox AH, Chess A and Lawrence JB: An architectural role for a nuclear noncoding RNA: NEAT1 RNA is essential for the structure of paraspeckles. Mol Cell 33: 717-726, 2009.

13. He C, Jiang B, Ma J and Li Q: Aberrant NEAT1 expression is associated with clinical outcome in high grade glioma patients. APMIS 124: 169-174, 2016.

14. Zeng $\mathrm{C}, \mathrm{Xu} \mathrm{Y,Xu} \mathrm{L,} \mathrm{Yu} \mathrm{X,} \mathrm{Cheng} \mathrm{J,} \mathrm{Yang} \mathrm{L,} \mathrm{Chen} \mathrm{S}$ and Li Y: Inhibition of long non-coding RNA NEAT1 impairs myeloid differentiation in acute promyelocytic leukemia cells. BMC Cancer 14: 693, 2014.

15. Sun C, Li S, Feng Z, Xi Y and Li D: Long non-coding RNA NEAT1 promotes non-small cell lung cancer progression through regulation of miR-377-3p-E2F3 pathway. Oncotarget 7: 51784-51814, 2016.

16. Zhu L, Yang N, Li C, Liu G, Pan W and Li X: Long noncoding RNA NEAT1 promotes cell proliferation, migration, and invasion in hepatocellular carcinoma through interacting with miR-384. J Cell Biochem 120: 1997-2006, 2018. 
17. Jovanovic $M$ and Hengartner MO: miRNAs and apoptosis: RNAs to die for. Oncogene 25: 6176-6187, 2006.

18. Shamsizadeh S, Goliaei S and Moghadam ZR: CAMIRADA Cancer microRNA association discovery algorithm, a case study on breast cancer. J Biomed Inform 94: 103180, 2019.

19. Kogure A, Kosaka N and Ochiya T: Cross-talk between cancer cells and their neighbors via miRNA in extracellular vesicles: An emerging player in cancer metastasis. J Biomed Sci 26: 7, 2019.

20. BožinovićK, SabolI, Dediol E, Milutin Gašperov N, Manojlović S, Vojtechova Z, Tachezy R and Grce M: Genome-wide miRNA profiling reinforces the importance of miR-9 in human papillomavirus associated oral and oropharyngeal head and neck cancer. Sci Rep 9: 2306, 2019.

21. Xiao F, Zhang W, Chen L, Chen F, Xie H, Xing C, Yu X, Ding S, Chen K, Guo H, et al: MicroRNA-503 inhibits the G1/S transition by downregulating cyclin D3 and E2F3 in hepatocellular carcinoma. J Transl Med 11: 195, 2013.

22. Xiao Y, Tian Q, He J, Huang M, Yang C and Gong L: miR-503 inhibits hepatocellular carcinoma cell growth via inhibition of insulin-like growth factor 1 receptor. Onco Targets Ther 9: 3535-3544, 2016.

23. Xie J, Murone M, Luoh SM, Ryan A, Gu Q, Zhang C, Bonifas JM, Lam CW, Hynes M, Goddard A, et al: Activating Smoothened mutations in sporadic basal-cell carcinoma. Nature 391: 90-92, 1998.

24. Wang Y, Zhou Z, Walsh CT and Mcmahon AP: Selective translocation of intracellular Smoothened to the primary cilium in response to Hedgehog pathway modulation. Proc Natl Acad Sci USA 106: 2623-2628, 2009.

25. Livak KJ and Schmittgen TD: Analysis of relative gene expression data using real-time quantitative PCR and the 2(-Delta Delta C(T))method. Methods 25: 402-408, 2001.

26. Li JH, Liu S, Zhou H, Qu LH and Yang JH: Starbase v2.0 Decoding miRNA-ceRNA, miRNA-ncRNA and protein-RNA interaction networks from large-scale clip-seq data. Nucleic Acids Res 42 (Database Issue): D92-D97, 2014.
27. Agarwal V, Bell GW, Nam JW and Bartel DP: Predicting effective microRNA target sites in mammalian mRNAs. Elife 4: e05005, 2015.

28. Du Z, Sun T, Hacisuleyman E, Fei T, Wang X, Brown M, Rinn JL, Lee MG, Chen Y, Kantoff PW and Liu XS: Integrative analyses reveal a long noncoding RNA-mediated sponge regulatory network in prostate cancer. Nat Commun 7: 10982, 2016.

29. Shao Y, Ye M, Li Q, Sun W, Ye G, Zhang X, Yang Y, Xiao B and Guo J: LncRNA-RMRP promotes carcinogenesis by acting as a miR-206 sponge and is used as a novel biomarker for gastric cancer. Oncotarget 7: 37812-37824, 2016.

30. Bruix J, Qin S, Merle P, Granito A, Huang YH, Bodoky G, Pracht M, Yokosuka O, Rosmorduc O, Breder V, et al: Regorafenib for patients with hepatocellular carcinoma who progressed on sorafenib treatment (RESORCE): A randomised, double-blind, placebo-controlled, phase 3 trial. Lancet 389: 56-66, 2017.

31. Xie Q, Lin S, Zheng M, Cai Q and Tu Y: Long noncoding RNA NEAT1 promotes the growth of cervical cancer cells via sponging miR-9-5p. Biochem Cell Biol 97: 100-108, 2018.

32. Wang L, Duan W, Kang L, Mao J, Yu X, Fan S, Li L and Tao Y: Smoothened activates breast cancer stem-like cell and promotes tumorigenesis and metastasis of breast cancer. Biomed Pharmacother 68: 1099-1104, 2014.

33. Walter K, Omura N, Hong SM, Griffith M, Vincent A, Borges M and Goggins M: Overexpression of smoothened activates the sonic hedgehog signaling pathway in pancreatic cancer-associated fibroblasts. Clin Cancer Res 16: 1781-1789, 2010.

This work is licensed under a Creative Commons Attribution-NonCommercial-NoDerivatives 4.0 International (CC BY-NC-ND 4.0) License. 